\title{
SARS-CoV-2 infection presenting as sustained atrial flutter and advanced ventricular dysfunction
}

\author{
Jennifer A Su*, Molly A Weisert, Michael J Silka, Yaniv Bar-Cohen and JonDavid Menteer \\ Division of Cardiology, Department of Pediatrics, Children's Hospital Los Angeles, Los Angeles, CA, USA
}

\begin{abstract}
We report a case of a 15-year-old girl who presented with atrial flutter and acute decompensated heart failure ultimately requiring cardiac support with extracorporeal membrane oxygenation (ECMO). While the patient's atrial flutter and cardiac dysfunction are believed to be related, the spontaneous development of atrial flutter is exceedingly rare in teenagers. While both arrhythmia and decompensated heart failure can be seen in fulminant myocarditis, the patient did not have a clinical history or testing consistent with myocarditis or systemic inflammation.

Upon her admission to the intensive care unit, her nasopharyngeal SARS-CoV-2 PCR was negative. However, her anti-SARS-CoV-2 IgG antibody titer was mildly elevated upon admission and increased four-fold within a month during her hospitalization, suggesting recent infection. After 8 days of ECMO support, the patient's heart function improved, and she was successfully decannulated. She later underwent an electrophysiology study with successful ablation of atrial flutter. She has not experienced further atrial arrhythmias and was ultimately discharged 2 months later with normal ventricular function by echocardiogram. Throughout her lengthy hospitalization she underwent numerous tests to elucidate her unusual clinical picture. Remote COVID-19 infection remains the most likely explanation for the timing and severity of this patient's clinical course.
\end{abstract}

\begin{abstract}
Abbreviations: COVID-19: Coronavirus disease of 2019; ECMO: Extracorporeal membrane oxygenation; EF: Ejection fraction; ICU: Intensive care unit; LVIDd: Left ventricular internal dimension in diastole; MIS-C: Multi-system inflammatory syndrome in children; MRI: Magnetic resonance imaging; SARS-CoV-2: Severe acute respiratory syndrome-coronavirus-2; TIC: Tachycardia induced cardiomyopathy.
\end{abstract}

\section{Introduction}

Viral infections may affect the cardiovascular system in various ways. A number of viruses are known to cause myocarditis and pericarditis, which may result in severe impairment of ventricular function leading to decompensated heart failure [1]. Viral infections may also cause systemic inflammatory response syndrome and septic shock from exaggerated immune inflammatory response [2]. Certain viruses have also been linked to development of cardiac arrhythmias $[3,4]$. In these cases, evidence of myocardial inflammation is often found. However, arrhythmias are sometimes the only indicator of cardiac involvement in viral infections. Additionally, myocardial failure of any cause can raise atrial and ventricular filling pressures, resulting in increased myocardial fiber stress, potentially inducing arrhythmias or triggering arrhythmias.

The coronavirus disease of 2019 (COVID-19), caused by severe acute respiratory syndrome-coronavirus-2 (SARS-CoV-2), became a worldwide pandemic in March 2020. This disease has already had devastating effects on the adult population, accounting for over 1.5 million fatalities worldwide [5]. Most frequent causes of morbidity and mortality are due to septic shock and respiratory failure [6]. Cardiovascular involvement, such as myocarditis and arrhythmias, is also common [7]. In pediatrics, however, morbidity and mortality from SARS-CoV-2 appears to be attenuated. Much of the current focus of SARS-CoV-2 in pediatrics, in fact, is on its latent effects of multisystem inflammatory syndrome in children (MIS-C) which is a toxic shock-like syndrome [8]. We report here our cautionary experience of a latent SARS-CoV-2 infection complication causing atrial arrhythmia, decompensated heart failure, and severe hemodynamic compromise requiring extracorporeal membrane oxygenation (ECMO) support before cardiac recovery 2 weeks later.

\section{Case report}

Our patient is a 15 -year-old female with a history of scoliosis, for which she had undergone spinal fusion 8 months prior to presentation. She was brought to the emergency department with a one-day history of shortness of breath and orthopnea. She denied any preceding palpitations, chest pain, viral symptoms, fever, or changes in energy level, and she had no known ill contacts. Initial vital signs revealed tachycardia with a heart rate of $150 \mathrm{bpm}$, and hypoxemia with an oxygen saturation of $85 \%$. Her chest radiograph was significant for a moderately enlarged heart, patchy infiltrates seen in the left lower lobe, with a trace left-sided pleural effusion. Her laboratory data revealed an elevated brain natriuretic peptide level of $1,503 \mathrm{pg} / \mathrm{mL}$, with normal transaminase, blood urea nitrogen and creatinine levels. Complete blood count revealed total leukocyte count of $8.04 \mathrm{~K} / \mu \mathrm{L}(62 \%$

${ }^{\star}$ Correspondence to: Jennifer A Su, Division of Cardiology, Department of Pediatrics, Children's Hospital Los Angeles, Los Angeles, CA, USA, Tel: +13233615136; E-mail: jsu@chla.usc.edu

Key words: adolescent, atrial flutter, COVID-19, ECMO, heart failure, myocarditis, SARS-CoV-2, ventricular dysfunction

Received: December 08, 2020; Accepted: December 28, 2020; Published: December 31, 2020 
neutrophils, $26 \%$ lymphocytes, $10 \%$ monocytes) with normal platelet count and hematocrit. Erythrocyte sedimentation rate and C-reactive protein were within laboratory specific normal limits. A blood culture drawn on presentation remained negative. A respiratory viral panel by PCR was negative for adenovirus, coronavirus, metapneumovirus, rhinovirus, enterovirus, influenza, parainfluenza, and respiratory syncytial virus. Blood PCR studies were negative for adenovirus, cytomegalovirus, enterovirus, Epstein Barr virus, human herpes virus 6, herpes simplex virus, parvovirus, coxsackie virus, echovirus, human immunodeficiency virus; and nasopharyngeal PCR for SARS-CoV-2 was negative on two occasions. The Troponin-I level was normal. Echocardiography demonstrated severely decreased left ventricular function with an ejection fraction (EF) of $22 \%$, without significant ventricular dilation (LVIDd $5.3 \mathrm{~cm}$, Z-score +1.4 standard deviation above the mean). Electrocardiogram revealed atrial flutter with 2:1 atrio-ventricular conduction at $148 \mathrm{bpm}$ (Figure 1). She was transferred to the cardiothoracic intensive care unit (ICU) for further management of newly diagnosed arrhythmia and decompensated heart failure.

In the ICU, the decision was made to intubate and sedate the patient for DC cardioversion. Upon anesthesia induction, she became hypotensive and bradycardic, then asystolic followed by a wide QRS rhythm which quickly degenerated to ventricular fibrillation. She was successfully defibrillated and intubated, but remained hemodynamically labile, with recurrent ventricular arrhythmias ultimately requiring cannulation for veno-arterial ECMO support.

The patient remained on ECMO support for 8 days. A subsequent echocardiogram obtained on day 13 of hospitalization revealed normalized left ventricular systolic function with an EF of $60 \%$ while on inotropic infusion (milrinone $0.5 \mathrm{mcg} / \mathrm{kg} / \mathrm{min}$, dopamine $2.5 \mathrm{mcg} / \mathrm{kg} / \mathrm{min}$, epinephrine $0.02 \mathrm{mcg} / \mathrm{kg} / \mathrm{min}$ ). These inotropic agents were successfully weaned off 4 days later (hospital day 17), and cardiac contractility remained normal. The patient required inpatient rehabilitation for 11 days, and she was discharged home from the hospital 2 months after initial presentation. She subsequently underwent electrophysiology study with an inducible cavo-tricuspid isthmus-dependent (atypical clockwise) atrial flutter, which was successfully ablated. Her cardiac systolic function has remained normal without recurrence of arrhythmia.

An extensive infectious evaluation was completed to identify the etiology of presumed myocarditis. Endomyocardial biopsy obtained at the time of ECMO decannulation showed no evidence of myocardial necrosis, inflammation, or active infection. The tissue sample was also tested for SARS-CoV-2 PCR and was also negative. A cardiac magnetic resonance imaging (MRI) was also obtained after ECMO decannulation, which included quantitative T1 and T2 mapping suggestive of elevated extracellular volume (37\%) without late phase enhancement, consistent with nonspecific myocardial edema and/or inflammation. Although her blood and respiratory viral PCR studies were negative from nasopharyngeal samples, an anti-SARS-CoV-2 IgG antibody titer was weakly positive upon admission $(0.8$; normal $<0.7$, borderline $0.8-1.1)$, 1.3 at 2 weeks, and 5.4 at 6 weeks (SARS-CoV-2 IgG ELISA developed for clinical use by Diagnostic Immunology Laboratory at Children's Hospital Los Angeles, Department of Pathology \& Laboratory Medicine). Anti-SARS-CoV-2 IgM antibody testing was not obtained. All other infectious and metabolic labs remained negative.

\section{Discussion and Conclusion}

Our case is unusual in that although COVID-19 has been linked to fulminant myocarditis in numerous reports, our patient did not demonstrate any evidence of active infection or inflammation. She had no history of recent fever or infectious illness, had no elevation of inflammatory markers, no detectable active infection upon laboratory testing, and no inflammation on endomyocardial biopsy. She had no known contact with a person known to have contracted COVID-19. Based on the lack of evidence for myocardial injury, and the electrophysiologic finding of a substrate for atrial flutter, it is possible that the patient's acute decompensated heart failure was caused, at least in part, by sustained atrial flutter rather than myocarditis. Three scenarios were possible: 1.) SARS-CoV-2 infection resulted in the onset of atrial flutter, with secondary development of tachycardia induced cardiomyopathy (TIC); 2.) COVID-19 caused myocarditis with hemodynamic and electrophysiologic changes resulting in atrial flutter; and 3.) COVID-19 resulted directly in the development of both atrial flutter and myocarditis in the weeks prior to hospitalization, with the combined effects of atrial flutter and COVID-19 resulting in impaired ventricular function.

Tachycardia induced cardiomyopathy (TIC) is associated with a number of tachyarrhythmias. In adults, it is diagnosed frequently in the setting of atrial fibrillation and flutter, but is less often encountered in the pediatric population because these tachyarrhythmias do not occur with frequency in children or adolescents [9]. TIC can develop as early as 3 days after onset of tachyarrhythmia, and ventricular dysfunction is often rapidly reversible with appropriate treatment of the arrhythmia [9]. Despite having an abnormally high ventricular heart rate of 150 bpm, our patient did not experience any palpitations, which made it challenging to determine the onset and duration of her atrial flutter. Her rapid cardiac recovery with essentially normal ventricular function two weeks following defibrillation is consistent with the recovery timeline expected of TIC.

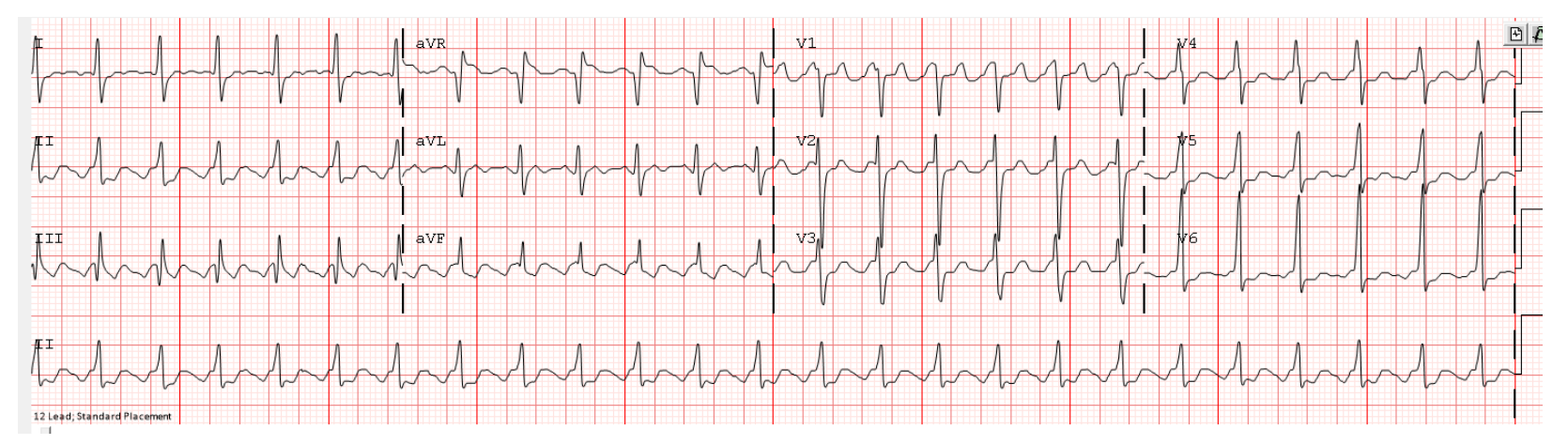

Figure 1. Patient's electrocardiogram obtained upon admission, demonstrating atrial flutter with $2: 1$ atrio-ventricular conduction at 148 bpm 
As part of the evaluation for myocarditis, our patient underwent a battery of infectious tests, all of which were negative for active infection. The patient's cardiac MRI did reveal myocardial edema and inflammation, but interpretation is difficult as the patient had undergone cardiopulmonary resuscitation, defibrillation, and ECMO cannulation shortly before MRI imaging. Although the COVID-19 PCR was negative, an anti-SARS-CoV IgG titer was found to be mildly positive in the early phase of her hospital admission. Repeat testing two- and six-weeks later revealed steady increase in SARS-CoV IgG antibodies, indicating that our patient had likely been infected approximately two to three weeks prior to her acute cardiac presentation [10].

Based on the timeline of our patient's clinical course and the results of tests performed, it seems most likely that our patient suffered a SARS-CoV-2 infection in the weeks prior to hospital admission, which presented with atrial flutter and ventricular dysfunction. While it may be possible that our patient had frank COVID-19 myocarditis manifesting both with arrhythmia and cardiac dysfunction, the absence of inflammation or myocardial damage, and her rapid recovery within two weeks after DC cardioversion are unusual for myocarditis [11]. However, as SARS-CoV-2 is a relatively novel virus, there remains much to be learned, including the expected clinical timeline of SARS$\mathrm{CoV}-2$ infection as it relates to the heart, particularly in pediatrics.

The majority of current literature and understanding of COVID-19 clinical repercussions draws from adult experience, and much of the pediatric experience with SARS-CoV-2 remains within the context of MIS-C. Continued reporting of unique clinical manifestations of this virus is essential to refine the medical knowledge of our current pandemic.

\section{Funding}

None.

\section{Financial disclosure}

The authors have no financial relationships relevant to this article to disclose.

\section{Competing interests}

The authors have no conflicts of interest to disclose.

\section{Acknowledgements}

\section{None.}

\section{References}

1. Mahrholdt H, Wagner A, Deluigi CC, Kispert E, Hager S, et al. (2006) Presentation, patterns of myocardial damage, and clinical course of viral myocarditis. Circulation 114: 1581-1590. [Crossref]

2. Lin GL, McGinley JP, Drysdale SB, Pollard AJ (2018) Epidemiology and immune pathogenesis of viral sepsis. Front Immunol 9: 2147. [Crossref]

3. Baksi AJ, Kanaganayagam GS, Prasad SK (2015) Arrhythmias in viral myocarditis and pericarditis. Card Electrophysiol Clin 7: 269-281. [Crossref]

4. Sardana M, Hsue PY, Tseng ZH, Vittinghoff E, Nah G, et al. (2019) Human immunodeficiency virus infection and incident atrial fibrillation. $\mathrm{J} \mathrm{Am} \mathrm{Coll} \mathrm{Cardiol} \mathrm{74:}$ 1512-1514. [Crossref]

5. https://covid19.who.int/

6. Wu Z, McGoogan JM (2020) Characteristics of and important lessons from the coronavirus disease 2019 (COVID-19) outbreak in China: Summary of a report of 72314 cases from the Chinese center for disease control and prevention. JAMA 323: 1239-1242. [Crossref]

7. Kochi AN, Tagliari AP, Forleo GB, Fassini GM, Tondo C (2020) Cardiac and arrhythmic complications in patients with COVID-19. J Cardiovasc Electrophysiol 31: 1003-1008. [Crossref]

8. Feldstein LR, Rose EB, Horwitz SM, Collins JP, Newhams MM, et al. (2020) Multisystem inflammatory syndrome in U.S. children and adolescents. $N$ Engl J Med 383: 334-346. [Crossref]

9. Huizar JF, Ellenbogen KA, Tan AY, Kaszala K (2019) Arrhythmia-induced cardiomyopathy: JACC State-of-the-art review. J Am Coll Cardiol 73: 2328-2344. [Crossref]

10. Jacofsky D, Jacofsky EM, Jacofsky M (2020) Understanding antibody testing for COVID-19. J Arthroplasty 35: S74-S81. [Crossref]

11. Felker GM, Boehmer JP, Hruban RH, Hutchins GM, Kasper EK, et al. (2000) Echocardiographic findings in fulminant and acute myocarditis. J Am Coll Cardiol 36 : 227-232. [Crossref]

Copyright: (C2020 Su JA. This is an open-access article distributed under the terms of the Creative Commons Attribution License, which permits unrestricted use, distribution, and reproduction in any medium, provided the original author and source are credited. 